ARTIFICIAL SATELLITES, Vol. 42, No. 1 - 2007

DOI: 10.2478/v10018-007-0015-3

\title{
LOCAL ORBITAL DERIVATIVES OF THE EARTH POTENTIAL EXPRESSED IN TERMS OF THE SATELLITE CARTESIAN COORDINATES AND VELOCITY
}

\author{
M. S. Petrovskaya, A. N. Vershkov \\ Central (Pulkovo) Astronomical Observatory of the Russian Academy of Sciences \\ Pulkovskoe Shosse 65/1, St. Petersburg, 196140, Russia \\ e-mail: petrovsk@gao.spb.ru \\ Tel.: +7-812-7234422, Fax:+7-812-3143360
}

\begin{abstract}
In a satellite gradiometry mission the observables will be the second order derivatives of the Earth potential in the local orbital reference frame. The conventional expansions for these derivatives contain singular factors. They depend on the functions of the orbit inclination $I$ and their first and second order derivatives. If the orbit eccentricity is taken into account then the functions of the eccentricity also involve these expressions. In the present paper more simple alternative expansions for the orbital derivatives are constructed, depending on the spherical coordinates and $\cos I$. They have only two sums and do not have singular factors. These expansions depend on the Legendre functions of the latitude but do not depend on their derivatives. As compared to the earlier expressions of the authors the present ones have the form which is more convenient for computations. Besides, these expressions can be applied not only for the case when the satellite orbit is circular and $\pi / 2 \leq I \leq \pi$ but for the arbitrary eccentricity $(0 \leq e<1)$ and inclination $(0 \leq I \leq \pi)$. After additional transformations the final expansions for the orbital derivatives represent, for the first time, simple functions of the cartesian coordinates of the satellite and the components of its velocity. These expressions may be convenient for inverting a huge amount of the GOCE data in the geopotential coefficients.
\end{abstract}

Keywords: local orbital derivatives - arbitrary satellite orbits - spherical and cartesian coordinates - geopotential model - GOCE satellite gradiometry mission

\section{INTRODUCTION}

The first satellite gradiometry mission GOCE of the European Space Agency (ESA) will start in 2007. In this mission the second order derivatives of the Earth potential in the local orbital reference frame will be measured. On the basis of these data a new high precision model of the geopotential will be constructed. For compiling observation equations various forms of expressions for the orbital derivatives are developed. These results are thoroughly outlined by Rummel et al. (1993) and Koop (1993). Basic principles of the time-wise method for solving the observation equations have been successfully developed in Klees et al. (2000a), Sneeuw (1992, 2000), Sneeuw et al. (2001).

In the time-wise approach to constructing a geopotential model from the GOCE mission data, developed in Rummel et al. (1993), Koop (1993), ESA (2003), the expansions for the 
orbital derivatives depend on the osculating Keplerian elements. These expressions involve the inclination functions and their first and second order derivatives. If the orbit eccentricity is not neglected then they depend also on the eccentricity functions (Kaula, 1966) which are different for the short-period and long-period perturbations. In publications of researches from Delft University, in particular Ditmar et al. (2003, p. 69), a conclusion is drawn that "the time-wise approach, when implemented correctly (i.e. without various approximations) is rather time-consuming technique and is hardly applicable to the inversion of the entire set of SGG data to be collected by GOCE."

In this connection it is a matter of much interest to develop alternative expressions for the orbital derivatives which have no deficiencies of the conventional expansions.

Such expressions are derived in Petrovskaya and Vershkov (2006). They represent double series depending on the spherical coordinates and the satellite track azimuth $\alpha$. These series do not contain any derivatives and have no singular factors. However, only the case is considered when $e=0$ and $\pi / 2 \leq I \leq \pi$. Different expressions for $\alpha$ are given, however without their derivation.

In the present paper the expansions for the orbital derivatives from (ibid.) are transformed to the form which simplifies the construction of a geopotential model from the gradiometry data. Now the general case is considered when there are no restrictions with respect to the values of the orbit eccentricity and inclination.

At first the orbital derivatives are presented in terms of the spherical coordinates and cos $I$. Then these expansions are transformed, for the first time, to simple expressions which depend only on the cartesian coordinates of the satellite and the components of its velocity.

\section{CONVENTIONAL AND NEW EXPRESSIONS FOR THE LOCAL NORTH-ORIENTED DERIVATIVES}

We consider the Earth's disturbing potential

$$
T=V-U,
$$

where $V$ represents the true gravitational potential and $U$ is the normal or another reference potential.

A truncated spherical harmonic series for $T$ has the form

$$
T=\frac{\mu}{a} \sum_{n=2}^{N} \sum_{|m|=0}^{n}\left(\frac{a}{r}\right)^{n+1} \bar{C}_{n, m} \bar{Y}_{n, m}(\theta, \lambda)
$$

where $\bar{Y}_{n, m}$ are the surface spherical harmonics

$$
\bar{Y}_{n, m}(\theta, \lambda)=\bar{P}_{n,|m|}(\cos \theta) Q_{m}(\lambda), Q_{m}(\lambda)=\left\{\begin{array}{l}
\cos m \lambda, m \geq 0 \\
\sin |m| \lambda, m<0 .
\end{array}\right.
$$

In (1) - (2) $r$ is the geocentric distance, $\theta$ and $\lambda$ are the colatitude and the longitude. The quantity $\mu=G M$ is the gravitational constant multiplied by the Earth's mass, $a$ is the semimajor axis of the reference ellipsoid, $\bar{C}_{n, m}$ and $\bar{P}_{n,|m|}(\cos \theta)$ represent the fully normalized harmonic coefficients and the associated Legendre functions, respectively. In (1) $m$ have positive and negative values. 


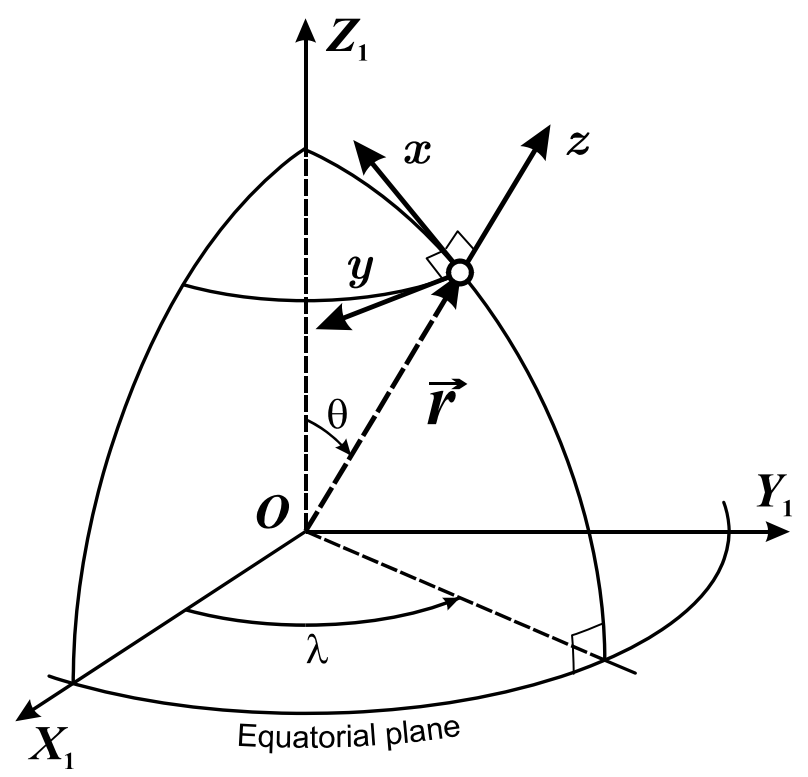

Fig. 1. Global reference frame $\left\{X_{1}, Y_{1}, Z_{1}\right\}$ and local north-oriented triad $\{x, y, z\}$

In Fig. 1 two reference frames are presented. The first of them is the global Earth-fixed coordinate system $\left\{X_{1}, Y_{1}, Z_{1}\right\}$ where $O$ is the Earth center, axis $Z_{1}$ coincides with the axis of the Earth rotation, axis $X_{1}$ is directed along the line of intersection of the Greenwich meridian (where $\lambda=0$ ) with the equatorial plane and $Y_{1}$ is directed to the East.

The second system is the local north-oriented reference frame $\{x, y, z\}$ in which $z$ has the geocentric radial direction, $x$ points to the north, and $y$ is directed to the west (a right-handed system).

There are the following expressions for the second order derivatives of the potential $T$ (Reed, 1973)

$$
\left.\begin{array}{l}
T_{z z}=T_{r r} \\
T_{x x}=\frac{1}{r} T_{r}+\frac{1}{r^{2}} T_{\theta \theta} \\
T_{y y}=\frac{1}{r} T_{r}+\frac{1}{r^{2} \tan \theta} T_{\theta}+\frac{1}{r^{2} \sin ^{2} \theta} T_{\lambda \lambda} \\
T_{x y}=\frac{1}{r^{2} \sin \theta} T_{\theta \lambda}-\frac{\cos \theta}{r^{2} \sin ^{2} \theta} T_{\lambda} \\
T_{x z}=\frac{1}{r^{2}} T_{\theta}-\frac{1}{r} T_{r \theta} \\
T_{y z}=\frac{1}{r^{2} \sin \theta} T_{\lambda}-\frac{1}{r \sin \theta} T_{r \lambda} .
\end{array}\right\}
$$

From the series (1) the expansion for the radial derivative follows

$$
T_{z z}=\frac{\mu}{a_{0}^{3}} \sum_{n=2}^{N} \sum_{m=-n}^{n}(n+1)(n+2)\left(\frac{a_{0}}{r}\right)^{n+3} \bar{C}_{n, m} \bar{Y}_{n, m}(\theta, \lambda) .
$$

By substituting the expressions for the other derivatives of $T$ with respect to $r, \theta$, $\lambda$ in the right-hand sides of equations (3) the conventional series for the north-oriented derivatives are obtained 


$$
\left.\begin{array}{l}
T_{x x}=\frac{\mu}{a^{3}} \sum_{n=2}^{N} \sum_{m=-n}^{n}\left(\frac{a}{r}\right)^{n+3} \bar{C}_{n, m}\left[-(n+1) \bar{P}_{n,|m|}(\cos \theta)-\right. \\
\left.+\frac{d^{2} \bar{P}_{n,|m|}(\cos \theta)}{d \theta^{2}}\right] Q_{m}(\lambda), \\
T_{y y}=\frac{\mu}{a^{3}} \sum_{n=2}^{N} \sum_{m=-n}^{n}\left(\frac{a}{r}\right)^{n+3} \bar{C}_{n, m}\left[-(n+1) \bar{P}_{n,|m|}(\cos \theta)-\right. \\
\left.+\frac{\cos \theta}{\sin \theta} \frac{d \bar{P}_{n,|m|}(\cos \theta)}{d \theta}-\frac{m^{2}}{\sin ^{2} \theta} \bar{P}_{n,|m|}(\cos \theta)\right] Q_{m}(\lambda), \\
T_{x y}=\frac{\mu}{a^{3}} \sum_{n=2}^{N} \sum_{m=-n}^{n}\left(\frac{a}{r}\right)^{n+3} \bar{C}_{n,-m}\left[\frac{1}{\sin \theta} \frac{d \bar{P}_{n,|m|}(\cos \theta)}{d \theta}\right. \\
\left.-\frac{\cos \theta}{\sin ^{2} \theta} \bar{P}_{n,|m|}(\cos \theta)\right] Q_{m}(\lambda), m \neq 0, \\
T_{x z}=\frac{\mu}{a^{3}} \sum_{n=2}^{N} \sum_{m=-n}^{n}(n+2)\left(\frac{a}{r}\right)^{n+3} \bar{C}_{n, m} \frac{d \bar{P}_{n,|m|}(\cos \theta)}{d \theta} Q_{m}(\lambda), \\
T_{y z}=\frac{\mu}{a^{3}} \sum_{n=2}^{N} \sum_{m=-n}^{n}(n+2) m\left(\frac{a}{r}\right)^{n+3} \bar{C}_{n,-m} \frac{\bar{P}_{n,|m|}(\cos \theta)}{\sin \theta} Q_{m}(\lambda), m \neq 0 .
\end{array}\right\}
$$

Expressions (5) depend on the associated Legendre functions and also on their derivatives of the first and second order. The series for $T_{y y}, T_{x y}$ and $T_{y z}$ contain singular factors $\sin ^{-1} \theta$ and $\sin ^{-2} \theta$ which become infinite at the poles.

As follows from the theory of the potential, the derivatives of $T$ are bounded functions in the whole exterior space. According to Ferrer's equation (Ilk, 1983, p. 119, (z.1.3)) the expression for the Legendre function $\bar{P}_{n,|m|}(\cos \theta)$ contains the factor $\sin ^{|m|} \theta$. However this factor does not eliminate the singular factors in equations (5). In particular, the last terms in the square brackets of the expressions for derivatives $T_{y y}, T_{x y}$ retain the singular factor $\sin ^{-1} \theta$ for $|m|=1$ and all $n$, after substituting Ferrer's expressions for the Legendre functions. Therefore only transformations of the complete expressions in the square brackets of equations (5) can eliminate all the singularities. Such transformations are performed in Petrovskaya and Vershkov (2006).

In (ibid.) the series in (5) are converted into simple expansions depending on the spherical coordinates $r, \theta, \lambda$ and not containing the derivatives of the Legendre functions and singular factors. We shall transform the series (4) and the expressions for the other derivatives from (ibid.) to the form which is more convenient for computations.

The order of summations of the series from (ibid.) is interchanged and the north-oriented derivatives are presented as Fourier series with respect to the longitude $\lambda$ 


$$
\left.\begin{array}{l}
T_{z z}=\sum_{|m|=0}^{N} \sum_{n=\max \{2,|m|\}}^{N} \bar{C}_{n, m} Q_{m}(\lambda) f_{m, n, 0}(r, \theta) \\
T_{y y}=\sum_{|m|=0}^{N} \sum_{n=\max \{2,|m|\}}^{N} \bar{C}_{n, m} Q_{m}(\lambda) f_{m, n, 1}(r, \theta) \\
T_{x x}=\sum_{|m|=0}^{N} \sum_{n=\max \{2,|m|\}}^{N} \bar{C}_{n, m} Q_{m}(\lambda) f_{m, n, 2}(r, \theta) \\
T_{x y}=\sum_{|m|=1}^{N} \sum_{n=\max \{2,|m|\}}^{N} \bar{C}_{n, m} Q_{-m}(\lambda) f_{m, n, 3}(r, \theta) \\
T_{z z}=\sum_{|m|=0}^{N} \sum_{n=\max \{2,|m|\}}^{N} \bar{C}_{n, m} Q_{m}(\lambda) f_{m, n, 4}(r, \theta) \\
T_{y z}=\sum_{|m|=1}^{N} \sum_{n=\max \{2,|m|\}}^{N} \bar{C}_{n, m} Q_{-m}(\lambda) f_{m, n, 5}(r, \theta) .
\end{array}\right\}
$$

Functions $f_{n, m, k}(r, \theta)$ have the form

$$
f_{m, n, k}(r, \theta)=\sigma_{n}(r) \tilde{f}_{m, n, k}(\theta),
$$

where

$$
\sigma_{n}(r)=\frac{\mu}{a^{3}}(n+1)(n+2)\left(\frac{a}{r}\right)^{n+3} .
$$

It can be noted that the factor $(n+1)(n+2)$ in (8) partly counteracts the attenuation factor $(a / r)^{n+3}$.

In (7)

$$
\left.\begin{array}{rl}
\widetilde{f}_{m, n, 0}(\theta) & =\bar{P}_{n,|m|}(\cos \theta), \\
\widetilde{f}_{m, n, 1}(\theta)= & \widetilde{a}_{n, m} \bar{P}_{n,|m|-2}(\cos \theta)+\widetilde{b}_{n, m} \bar{P}_{n,|m|}(\cos \theta) \\
& +\widetilde{c}_{n, m} \bar{P}_{n,|m|+2}(\cos \theta), \\
\widetilde{f}_{m, n, 2}(\theta)= & -f_{m, n, 0}(\theta)+f_{m, n, 1}(\theta), \\
\widetilde{f}_{m, n, 3}(\theta)= & \widetilde{d}_{n, m} \bar{P}_{n-1,|m|-2}(\cos \theta)+\widetilde{g}_{n, m} \bar{P}_{n-1,|m|}(\cos \theta) \\
& +\widetilde{h}_{n, m} \bar{P}_{n-1,|m|+2}(\cos \theta), \\
\widetilde{f}_{m, n, 4}(\theta)= & \widetilde{\beta}_{n, m} \bar{P}_{n,|m|-1}(\cos \theta)+\widetilde{\gamma}_{n, m} \bar{P}_{n,|m|+1}(\cos \theta) \\
\widetilde{f}_{m, n, 5}(\theta)= & \widetilde{\mu}_{n, m} \bar{P}_{n-1,|m|-1}(\cos \theta)+\widetilde{v}_{n, m} \bar{P}_{n-1,|m|+1}(\cos \theta) .
\end{array}\right\}
$$

The numerical coefficients $\widetilde{a}_{n, m}, \widetilde{b}_{n, m}$, etc., in (9) are provided in Appendix A. They differ from the similar coefficients $a_{n, m}, b_{n, m}$, etc., from (ibid., Appendix A) only by the factor $(n+1)(n+2)$ in the denominators.

* The value of $a_{n, m}$ for $|m|=0,1$ is misprinted in (ibid.). It should be $a_{n, m}=0$ and not $a_{n, m}=70$. 
The new constants are bounded for any values of $m$ and $n$, as compared to some constants from (ibid.) which increase with enlarging $n$ and $|m|$.

In comparison to the conventional expansions (5), the expansions (6) do not contain singularities and the first and second order derivatives of the Legendre functions. As compared to the expressions for $T_{x x}$ in (ibid.) it is essentially simplified by applying the Laplace equation.

In the next Section we shall apply expressions (6) for deriving new expressions for the local orbital derivatives.

\section{CONVENTIONAL AND NEW EXPRESSIONS FOR THE LOCAL ORBITAL DERIVATIVES}

We consider the global reference frame (Kaula, 1966, Section 2.3) which is presented in Fig. 2 . It is the conventional inertial system of coordinates $\{X, Y, Z\}$ where axis $Z$ coincides with the axis of the Earth rotation $Z_{1}$, axis $X$ points to the vernal equinox $\gamma$ and $Y$ is directed to the East.

The angle between $X$ and $X_{1}$ is equal to the Earth argument of the longitude $\left(\theta_{G}\right)$.

On Fig. 2 the local orbital reference frame $\{u, v, w\}$ is also presented where axis $w$ coincides with $z$, axis $v$ is directed along the instantaneous angular momentum vector and $u$ complements the right-handed triad.

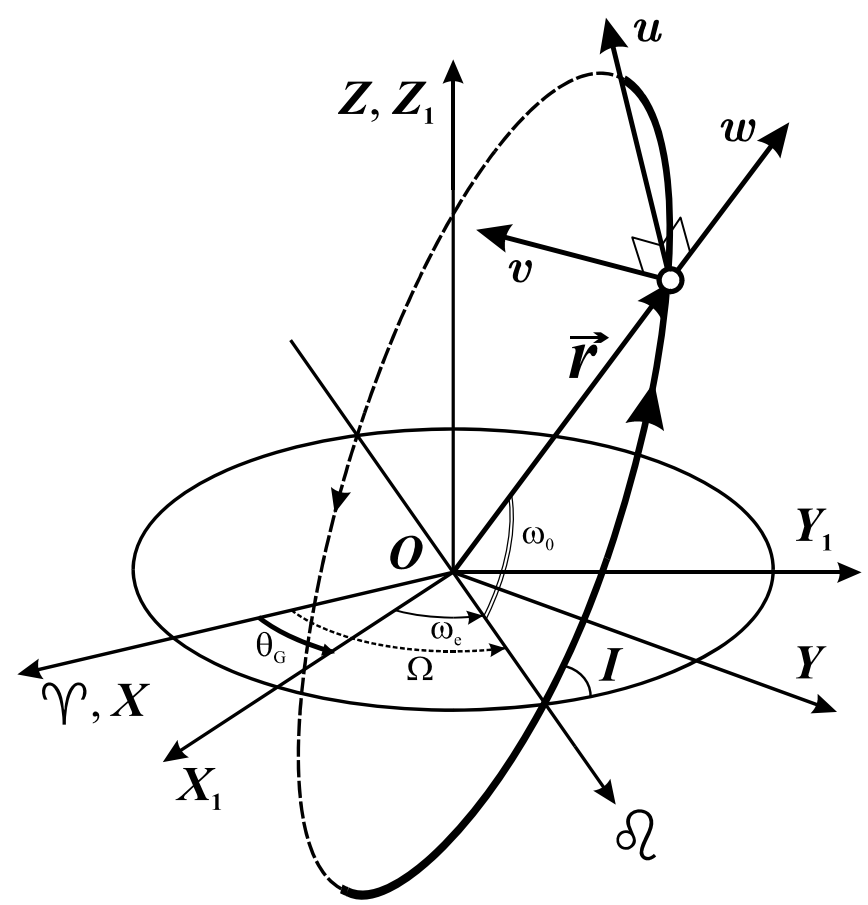

Fig. 2. Global reference frames $\{X, Y, Z\},\left\{X_{1}, Y_{1}, Z_{1}\right\}$ and local orbital triad $\{u, v, w\}$

When constructing a geopotential model from satellite gradiometry data by the time-wise approach the expressions for the orbital derivatives are considered as functions of time. In the conventional expressions they are presented in terms of $r$ and the osculating Keplerian elements $e, I, \Omega, \omega, M$ which are, respectively, the eccentricity, the inclination, the right ascension of the ascending node, the argument of the perigee and the mean anomaly. Due a small eccentricity of a satellite orbit, it is assumed in some theoretical and numerical 
investigations that $e=0$ (Rummel et al., 1993, p. 3.4), (Koop, 1993, p. 35) (Klees et al., 2000b, p.70).

In this case the potential $T$, defined in (1) and (2), is presented in the following form (Koop, 1993, pp. 35, 36)

$$
T=\sum_{l=0}^{N} \sum_{m=0}^{l} \sum_{k=-l}^{l} \frac{\mu}{a^{3}}\left(\frac{a}{r}\right)^{l+1} \bar{F}_{l m p}(I)\left[\alpha_{l m} \cos \psi_{k m}+\beta_{l m} \sin \psi_{k m}\right],
$$

where $p=\frac{l-k}{2}$ and index $k$ ranges from $-l$ to $l$ with a step-size 2 .

$\operatorname{In}(10)$

$$
\left.\begin{array}{l}
\psi_{k m}=k \omega_{0}+m \omega_{e} \\
\omega_{0}=\omega+M \\
\omega_{e}=\Omega-\theta_{G}
\end{array}\right\}
$$

There are the designations

$$
\begin{gathered}
\alpha_{l m}=\left\{\begin{array}{l}
\bar{C}_{l, m}, l-m: \text { even } \\
-\bar{S}_{n,|m|} l-m: \text { odd },
\end{array}\right. \\
\beta_{l m}=\left\{\begin{array}{c}
\bar{S}_{l, m}, l-m: \text { even } \\
-\bar{C}_{n, m} l-m: \text { odd },
\end{array}\right.
\end{gathered}
$$

where $\bar{C}_{l, m}$ corresponds to $\cos m \lambda$ and $\bar{S}_{l, m}$ to $\sin m \lambda$ in the spherical harmonic expansion for $T$.

In (10) $\bar{F}_{l, m, p}(I)$ are the normalized functions of the inclination $I$. According to Kaula (1966) and Rummel et al. (1993, p. A-3.7), these functions can be presented as follows

$$
\begin{aligned}
& \bar{F}_{l m p}(I)=\sum_{q=0}^{q_{\max }} \sum_{s=0}^{m} \sum_{c=c_{\min }}^{c_{\max }} \frac{(2 l-2 q) !(-1)^{j+c}}{2^{2 l-2 q} q !(l-q) !(l-m-2 q) !} \\
& \times\left(\begin{array}{c}
m \\
s
\end{array}\right)\left(\begin{array}{c}
l-m-2 q+s \\
c
\end{array}\right)\left(\begin{array}{c}
m-s \\
p-q-c
\end{array}\right) \sin ^{l-m-2 q} I \cos ^{s} I .
\end{aligned}
$$

Here

$$
q_{\max }=\min \{j, p\}, c_{\min }=\max \{0, p-q\}, c_{\max }=\min \{l-m-2 q+s, m-s\}
$$

and $j$ is the integer part of $\frac{l-m}{2}$.

The inclination functions (13) can be evaluated in different ways. More simple calculations are based on recurrent relations (Sneeuw, 1991).

In the conventional approach the orbital derivatives are considered in one of two systems of curvilinear coordinates (Rummel et al., 1993, pp. A-1.10, A-1.11). In one of them the coordinates $r, I, \omega_{0}$ are applied and in the other $r, \omega_{e}, \omega_{0}$.

In the first case there are the following expressions for the orbital derivatives 


$$
\left.\begin{array}{rl}
T_{u u} & =\frac{1}{r} T_{r}+\frac{1}{r^{2}} T_{\omega_{0} \omega_{0}} \\
T_{v v} & =\frac{\cos \omega_{0}}{r^{2} \sin \omega_{0}} T_{\omega_{0}}+\frac{1}{r} T_{r}+\frac{1}{r^{2} \sin ^{2} \omega_{0}} T_{I I} \\
T_{w w} & =T_{r r} \\
T_{u v} & =-\frac{\cos \omega_{0}}{r^{2} \sin ^{2} \omega_{0}} T_{I}+\frac{1}{r^{2} \sin \omega_{0}} T_{I \omega_{0}} \\
T_{u w} & =-\frac{1}{r^{2}} T_{\omega_{0}}+\frac{1}{r} T_{r \omega_{0}} \\
T_{v w} & =-\frac{1}{r^{2} \sin \omega_{0}} T_{I}+\frac{1}{r \sin \omega_{0}} T_{r I}
\end{array}\right\}
$$

and in the second case

$$
\left.\begin{array}{rl}
T_{u u}= & \frac{1}{r} T_{r}+\frac{1}{r 2} T_{\omega_{0} \omega_{0}} \\
T_{v v}= & \frac{1}{r} T_{r}+\frac{1}{r^{2} \sin ^{2} I \cos ^{2} \omega_{0}}\left[-\tan \omega_{0} \cos I T_{\omega_{e}}+T_{\omega e \omega e}+\tan \omega_{0}\left(\sin ^{2} I \sin ^{2} \omega_{0}\right.\right. \\
& \left.\left.-2 \sin ^{2} I+1\right) T_{\omega_{0}}-2 \cos I T_{\omega_{e} \omega_{0}}+\cos ^{2} I T_{\omega_{0} \omega_{0}}\right] \\
T_{\omega \omega}= & T_{r r} \\
T_{u v}= & -\frac{1}{r^{2} \sin I \cos \omega_{0}}\left[\tan \omega_{0} T_{\omega_{e}}-\tan \omega_{0} \cos I T_{\omega_{0}}+T_{\omega_{e} \omega_{0}}+\cos I T_{\omega_{0} \omega_{0}}\right] \\
T_{u w}= & \frac{1}{r} T_{r \omega_{0}}-\frac{1}{r^{2}} T_{\omega_{0}} \\
T_{v w}= & \frac{1}{r \sin I \cos \omega_{0}}\left[\frac{1}{r} T_{\omega_{e}}-\frac{\cos I}{r} T_{\omega_{0}}-T_{r \omega_{e}}+\cos I T_{r \omega_{0}}\right] .
\end{array}\right\}
$$

In the right-hand sides of equations (15) and (16) the partial derivatives of $T$ with respect $r$ and the orbital elements are substituted. They are found by differentiating the expressions (10) and (13) with respect to $r, I, \omega_{0}$ and $\omega_{e}$.

Let us note that the expressions (15) involve not only the inclination functions (13) but also their first and second order derivatives.

The initial series for the geopotential given in Kaula (1966) contains additional eccentricity functions, $G_{n, m, p}(e)$, in form of series depending on $e$. These functions have different form for the long and short period perturbations.

It is known (Pail and Plank, 2002) that in the GOCE mission the orbit eccentricity may be $e \approx 0.004$ and the variation of the inclination $\delta I<0.01^{\circ}$. Therefore the inclination functions vary with time (ibid., p. 466) and the eccentricity can not be neglected.

In the GOCE mission there will be several hundred millions of observations and 90000 of the unknown spherical harmonic coefficients of the geopotential (ibid., p. 462). Correspondingly, the solution of the observation equations will be very time-consuming (even for a circular satellite orbit), as follows from numerical studies of researches from Delft 
University. That is why an alternative approach is proposed (Ditmar and Klees, 2002), (Ditmar et al., 2003) in which the geopotential expansion (10) is not applied.

The expressions for three derivatives, $T_{v v}, T_{u v}$ and $T_{v w}$, have singularities: for $\omega_{0}=0, \pi$ in equations (15) and for $\omega_{0}=\pi / 2$ in equations (16). These singularities can be excluded by certain transformations (Rummel et al., 1993, p. 3.6). However the resulting expressions for the derivatives become more complicated than in (15) and (16) because they depend on the derivatives of $T$ entering the right-hand sides of both sets of equations.

An alternative approach is developed for eliminating the singularities (Rummel et al., 1993, pp. 3.7, 3.8). It is based on transferring from the equatorial reference plane for the latitudes to the orbital plane. However the corresponding expression for the modified inclination functions is more complicated than for the conventional functions in (13).

In Petrovskaya and Vershkov (2006) simple non-singular expressions are derived for the geopotential derivatives in the local orbital reference frame in the case of a circular satellite orbit. These expressions depend on the spherical coordinates and the satellite track azimuth. Several expressions for the azimuth are provided, without their derivation. In Appendix B we present this derivation and not only for the case when $e=0$ and $\pi / 2 \leq I \leq \pi$ but for the general case of an arbitrary eccentricity and inclination.

Besides, the expressions for the orbital derivatives from (ibid.) will be transformed to the form which is more convenient for processing satellite gradiometry data, when estimating the geopotential coefficients by the time-wise least squares adjustment.

In (ibid.) the orbital derivatives are presented as linear functions of the north-oriented derivatives

$$
\left.\begin{array}{l}
T_{u u}=-\cos ^{2} \alpha T_{z z}-\cos 2 \alpha T_{y y}+\sin 2 \alpha T_{x y} \\
T_{v v}=-\sin ^{2} \alpha T_{z z}+\cos 2 \alpha T_{y y}-\sin 2 \alpha T_{x y} \\
T_{u v}=\frac{1}{2} \sin 2 \alpha T_{z z}+\sin 2 \alpha T_{y y}+\cos 2 \alpha T_{x y} \\
T_{u w}=\cos \alpha T_{x z}+\sin \alpha T_{y z} \\
T_{v w}=-\sin \alpha T_{x z}+\cos \alpha T_{y z} \\
T_{w w}=T_{z z} .
\end{array}\right\}
$$

Here $\alpha$ is the satellite track azimuth, that is the angle between the local meridian in the observation point and the direction of axis $u$. This angle is reckoned from the north clockwise.

The expressions for $\alpha$ are given in (ibid.) for the case when $e=0$ and $\pi / 2 \leq I \leq \pi$. Now more general formulas for $a$ will be derived in Appendix B, corresponding to the case when $0 \leq e<1$ and $0 \leq I \leq \pi$.

The right-hand sides of equations (17) depend on five north-oriented derivatives, with excluding $T_{x x}$.

We substitute expressions (6) for the north-oriented derivatives in equations (17).

In the result, the expansions for the orbital derivatives are presented in the form of Fourier series with respect to $\lambda$ 


$$
\left.\begin{array}{l}
T_{w w}=\sum_{m=-N}^{N} \sum_{n=\max \{2,|m|\}}^{N} \bar{C}_{n, m} Q_{m}(\lambda) F_{m, n, 0}(r, \theta, \alpha) \\
T_{u u}=\sum_{m=-N}^{N} \sum_{n=\max \{2,|m|\}}^{N} \bar{C}_{n, m}\left[Q_{m}(\lambda) F_{m, n, 1}(r, \theta, \alpha)+Q_{-m}(\lambda) F_{m, n, 3}(r, \theta, \alpha)\right] \\
T_{v v}=\sum_{m=-N}^{N} \sum_{n=\max \{2,|m|\}}^{N} \bar{C}_{n, m}\left[Q_{m}(\lambda) F_{m, n, 2}(r, \theta, \alpha)-Q_{-m}(\lambda) F_{m, n, 3}(r, \theta, \alpha)\right] \\
T_{u v}=\sum_{m=-N}^{N} \sum_{n=\max \{2,|m|\}}^{N} \bar{C}_{n, m}\left[Q_{m}(\lambda) F_{m, n, 4}(r, \theta, \alpha)+Q_{-m}(\lambda) F_{m, n, 5}(r, \theta, \alpha)\right] \\
T_{u w}=\sum_{m=-N}^{N} \sum_{n=\max \{2,|m|\}}^{N} \bar{C}_{n, m}\left[Q_{m}(\lambda) F_{m, n, 6}(r, \theta, \alpha)+Q_{-m}(\lambda) F_{m, n, 7}(r, \theta, \alpha)\right] \\
T_{v w}=\sum_{m=-N}^{N} \sum_{n=\max \{2,|m|\}}^{N} \bar{C}_{n, m}\left[Q_{m}(\lambda) F_{m, n, 8}(r, \theta, \alpha)+Q_{-m}(\lambda) F_{m, n, 9}(r, \theta, \alpha)\right] .
\end{array}\right\}
$$

Here

$$
F_{m, n, k}(r, \theta, \alpha)=\sigma_{n}(r) \widetilde{F}_{m, n, k}(\theta, \alpha), k=0,1,2, \ldots, 9,
$$

where function $\sigma_{n}(r)$ is defined in (8).

Functions $\widetilde{F}_{m, n, k}(\theta, \alpha)$ have the following expressions

$$
\left.\begin{array}{l}
\widetilde{F}_{m, n, 0}(\theta, \alpha)=\widetilde{f}_{m \cdot n \cdot 0}(\theta) \\
\widetilde{F}_{m, n, 1}(\theta, \alpha)=-\cos ^{2} \alpha \widetilde{f}_{m, n, 0}(\theta)+\cos 2 \alpha \widetilde{f}_{m, n, 1}(\theta) \\
\widetilde{F}_{m, n, 2}(\theta, \alpha)=-\sin ^{2} \alpha \widetilde{f}_{m, n, 0}(\theta)-\cos 2 \alpha \widetilde{f}_{m, n, 1}(\theta) \\
\widetilde{F}_{m, n, 3}(\theta, \alpha)=\sin 2 \alpha \widetilde{f f}_{m, n, 3}(\theta) \\
\widetilde{F}_{m, n, 4}(\theta, \alpha)=\frac{1}{2} \sin 2 \alpha \widetilde{d f}_{m, n, 0}(\theta)-\sin 2 \alpha \widetilde{f}_{m, n, 1}(\theta) \\
\widetilde{F}_{m, n, 5}(\theta, \alpha)=\cos 2 \alpha \widetilde{f}_{m, n, 3}(\theta) \\
\widetilde{F}_{m, n, 6}(\theta, \alpha)=\cos \alpha \widetilde{f}_{m, n, 4}(\theta) \\
\widetilde{F}_{m, n, 7}(\theta, \alpha)=\sin \alpha \widetilde{f}_{m, n, 5}(\theta) \\
\widetilde{F}_{m, n, 8}(\theta, \alpha)=-\sin \alpha \widetilde{f}_{m, n, 4}(\theta) \\
\widetilde{F}_{m, n, 9}(\theta, \alpha)=\cos \alpha \widetilde{f}_{m, n, 5}(\theta) .
\end{array}\right\}
$$

Functions $\widetilde{f}_{m, n, k}(\theta)$ in the right-hand sides of (20) are defined in (9).

In each equation from (18) there are two sums. Besides, the right-hand sides of these equations do not depend on any derivatives and have no singularities. 


\section{ORBITAL DERIVATIVES AS FUNCTIONS OF THE SATELLITE POSITION AND THE VELOCITY}

In the conventional expressions for the orbital derivatives and in the expressions for them in Petrovskaya and Vershkov (2006) the case of a circular satellite orbit is considered, when $e=0$ and the argument of the latitude $\omega_{0}$ is equal to $\omega+M$, where $w$ is the argument of the perigee and $M$ is the mean anomaly. In the present paper we consider the general case when the satellite orbit has an arbitrary eccentricity $0 \leq e<1$. The corresponding argument of the latitude is equal to $\omega_{0}=\omega+v$, where $v$ is the true anomaly. Besides, the inclination is also arbitrary, that is $0 \leq I \leq \pi$.

There can be two approaches for choosing the angular variables in equations (18). In one of them these variables are $\omega_{0}$ and $\lambda$ and in the other case they are $\theta$ and $\lambda$. Correspondingly, in the first case the angles $\theta$ and $\alpha$ are expressed in terms of $\omega_{0}$ and in the second case $\alpha$ is a function of $\theta$.

In (ibid.) equations for the angular variables are given, in which it was assumed that $e=0$ and $\pi / 2 \leq I \leq \pi$.

In this Section we shall use the following expressions, derived in Appendix B,

$$
\begin{aligned}
& \sin \theta=\sqrt{1-\sin ^{2} I \sin ^{2} \omega_{0}}, \\
& \cos \theta=\sin I \sin \omega_{0}, \\
& \sin \alpha=\frac{\cos I}{\sqrt{1-\sin ^{2} I \sin ^{2} \omega_{0}}}, \\
& \cos \alpha=\frac{\sin I \cos \omega_{0}}{\sqrt{1-\sin ^{2} I \sin ^{2} \omega_{0}}}
\end{aligned}
$$

and

$$
\begin{aligned}
& \sin \alpha=\frac{\cos I}{\sin \theta}, \\
& \cos \alpha= \pm \frac{\sqrt{\sin ^{2} I-\cos ^{2} \theta}}{\sin \theta} .
\end{aligned}
$$

As was mentioned above, now $\omega_{0}=\omega+v .^{*}$

In (23) the 'plus' sign corresponds to the ascending satellite track and the 'minus' sign to the descending one.

Let us note that the first equation in (23) was given in Vermeer (1990), only without its derivation.

In the case when the angular variables are chosen to be $\theta$ and $\lambda$ it is convenient for computations to present (23) in a more simple form

\footnotetext{
* In (ibid.) the expression for $\cos \alpha$, depending on $\omega_{0}$, has a superfluous symbol \pm .
} 


$$
\begin{aligned}
& \sin \alpha=\frac{\cos I}{\sin \theta} \\
& \cos \alpha= \pm \sqrt{1-\sin ^{2} \alpha} .
\end{aligned}
$$

The most important advantage of (24) is that expressions (9) and hence (18) - (20) depend directly on $\theta$ and not on $\omega_{0}$. Therefore, if the time variable is $\omega_{0}$ then it would be necessary to transfer in the Legendre functions in equations (9) from the variable $\theta$ to the variable $\omega_{0}$, with the aid of (21).

Taking into account this remark, equations (24) can be recommended for the implementation.

The satellite in the GOCE mission will be continuously tracked with the aid of an onboard GPS/Glonass receiver which is used as a Satellite-to-Satellite Tracking Instrument (ESA, 2003). Thus, the satellite position and velocity at the moment of observations will be known. If functions $\theta, \lambda$ and $I$ are presented in terms of three cartesian coordinates and three components of the velocity then expressions (24) for the azimuth and expressions (18) - (20) for the orbital derivatives will become functions of these six variables.

According to Fig. 1, function $\cos \theta$ is presented in the form (Heiskanen and Moritz, 1967)

$$
\cos \theta=\frac{Z_{1}}{r}
$$

where

$$
r=\sqrt{X_{1}^{2}+Y_{1}^{2}+Z_{1}^{2}}
$$

From (25) and (26) follows

$$
\sin \theta=\frac{\sqrt{X_{1}^{2}+Y_{1}^{2}}}{r} .
$$

Equations (18) represent the Fourier series with respect to the longitude $\lambda$. The value of $\lambda$ is defined by the equation (ibid.)

$$
\lambda=\arctan \frac{Y_{1}}{X_{1}}
$$

Function $\cos I$ entering the first equation in (24) can be expressed in terms of coordinates $X, Y, Z$ (Fig. 2) and components $\dot{X}, \dot{Y}, \dot{Z}$ of the satellite velocity.

From the integrals of the areas (Smart, 1953, Section 2) follows that

$$
\cos I=\frac{C_{3}}{C}
$$

where

$$
\left.\begin{array}{l}
C_{1}=Y \dot{Z}-Z \dot{Y} \\
C_{2}=Z \dot{X}-X \dot{Z} \\
C_{3}=X \dot{Y}-Y \dot{X}
\end{array}\right\}
$$

and 


$$
C=\sqrt{C_{1}^{2}+C_{2}^{2}+C_{3}^{2}}
$$

After applying equations (24), (27) and (29) we obtain

$$
\sin \alpha=\frac{r}{\sqrt{X_{1}^{2}+Y_{1}^{2}}} \frac{C_{3}}{C} .
$$

Function $\cos \alpha$ is evaluated from (24).

Let us consider function $\sigma_{n}(r)$, entering (19). It is defined in (8).

The geocentric distance of the satellite is presented in the form

$$
r=r_{0}+\delta r
$$

where $r_{0}$ is the radius of a mean orbital sphere and $\delta r$ is the deviation of $r$ from $r_{0}$.

From (8) and (33) we can write

$$
\sigma_{n}(r)=\sigma_{n}\left(r_{0}\right) \omega_{n}(r)
$$

where

$$
\sigma_{n}\left(r_{0}\right)=\frac{\mu}{a^{3}}(n+1)(n+2)\left(\frac{a}{r_{0}}\right)^{n+3}
$$

and

$$
\omega_{n}(r)=\left(1+\frac{\delta r}{r_{0}}\right)^{-n-3}
$$

Function $\omega_{n}(r)$ can be expanded in the power series

$$
\omega_{n}(r)=1+\sum_{p=1}^{\infty} \nu_{p}(-1)^{p}\left(\frac{\delta r}{r_{0}}\right)^{p} .
$$

We consider a truncated series

$$
\omega_{n}(r)=1+\sum_{p=1}^{p_{\max }} \nu_{p}(-1)^{p}\left(\frac{\delta r}{r_{0}}\right)^{p}
$$

where the constants $v_{p}$ have the expressions

$$
v_{p}=\frac{(n+3)(n+4) \ldots(n+p+2)}{p !}
$$

In the GOCE mission $r_{0} \approx a+250 \mathrm{~km}$ and the quantity $\delta r / r_{0}$ in (36) will be small $\left(|\delta r| / r_{0} \approx 0.004\right)$. Then, with the accuracy $10^{-16}$, in the series (36) it can be taken $p_{\max }=20$.

Since the quantities $\sigma_{n}\left(r_{0}\right)$ in (35) and $v_{p}$ in (37) are constants then they can be calculated only one time and retained in the computer storage.

In the result, the orbital derivatives are determined by (9), (18) - (20), the second equation in (24) and (30) - (37). 
Equations (18) can be solved with respect to the potential coefficients $\bar{C}_{n, m}$ on the basis of gradiometry data by the least squares time-wise approach.

The same equations can be applied for simulating the orbital derivatives from a known geopotential model.

\section{FORMULAS FOR COMPUTATIONS}

We present the orbital derivatives as functions of the moments $t_{j}$ of the gradiometry measurements, where $j=1,2,3, \ldots, j_{\max }$.

For convenience of implementation all the necessary formulas are combined together.

Equations (18) are presented in the form

$$
\left.\begin{array}{l}
T_{w w}\left(t_{j}\right)=\sum_{m=-N} \sum_{n=\max \{2,|m|\}}^{N} \bar{C}_{n, m} Q_{m}\left(\lambda_{j}\right) \bar{F}_{m, n, 0}\left(t_{j}\right) \\
T_{u u}\left(t_{j}\right)=\sum_{m=-N}^{N} \sum_{n=\max \{2,|m|\}}^{N} \bar{C}_{n, m}\left[Q_{m}\left(\lambda_{j}\right) \bar{F}_{m, n, 1}\left(t_{j}\right)+Q_{-m}\left(\lambda_{j}\right) \bar{F}_{m, n, 3}\left(t_{j}\right)\right] \\
T_{v v}\left(t_{j}\right)=\sum_{m=-N}^{N} \sum_{n=\max \{2,|m|\}}^{N} \bar{C}_{n, m}\left[Q_{m}\left(\lambda_{j}\right) \bar{F}_{m, n, 2}\left(t_{j}\right)-Q_{-m}\left(\lambda_{j}\right) \bar{F}_{m, n, 3}\left(t_{j}\right)\right] \\
T_{u v}\left(t_{j}\right)=\sum_{m=-N}^{N} \sum_{n=\max \{2,|m|\}}^{N} \bar{C}_{n, m}\left[Q_{m}\left(\lambda_{j}\right) \bar{F}_{m, n, 4}\left(t_{j}\right)+Q_{-m}\left(\lambda_{j}\right) \bar{F}_{m, n, 5}\left(t_{j}\right)\right] \\
T_{u w}\left(t_{j}\right)=\sum_{m=-N}^{N} \sum_{n=\max \{2,|m|\}}^{N} \bar{C}_{n, m}\left[Q_{m}\left(\lambda_{j}\right) \bar{F}_{m, n, 6}\left(t_{j}\right)+Q_{-m}\left(\lambda_{j}\right) \bar{F}_{m, n, 7}\left(t_{j}\right)\right] \\
T_{v w}\left(t_{j}\right)=\sum_{m=-N}^{N} \sum_{n=\max \{2,|m|\}}^{N} \bar{C}_{n, m}\left[Q_{m}\left(\lambda_{j}\right) \bar{F}_{m, n, 8}\left(t_{j}\right)+Q_{-m}\left(\lambda_{j}\right) \bar{F}_{m, n, 9}\left(t_{j}\right)\right]
\end{array}\right\}
$$

From (19) and (34) follows

$$
\bar{F}_{m, n, k}\left(t_{j}\right)=\sigma_{n}\left(r_{0}\right) \omega_{n}\left(r_{j}\right) \widetilde{F}_{m, n, k}\left(\theta_{j}, \alpha_{j}\right), k=0,1,2, \ldots, 9 .
$$

The constants $\sigma_{n}\left(r_{0}\right)$ are defined in (35).

From (33) and (36) we have

$$
\omega_{n}\left(r_{j}\right)=1+\sum_{p=1}^{p_{\max }} v_{p}(-1)^{p}\left(\frac{\delta r_{j}}{r_{0}}\right)^{p}, \delta r_{j}=r_{j}-r_{0},
$$

where the constants $v_{p}$ are given in (37).

Equations (20) are written in the form 


$$
\left.\begin{array}{l}
\widetilde{F}_{m, n, 0}\left(\theta_{j}, \alpha_{j}\right)=\widetilde{f}_{m, n, 0}\left(\theta_{j}\right) \\
\widetilde{F}_{m, n, 1}\left(\theta_{j}, \alpha_{j}\right)=-\cos ^{2} \alpha_{j} \widetilde{f}_{m, n, 0}\left(\theta_{j}\right)+\cos 2 \alpha_{j} \widetilde{f}_{m, n, 1}\left(\theta_{j}\right) \\
\widetilde{F}_{m, n, 2}\left(\theta_{j}, \alpha_{j}\right)=-\sin ^{2} \alpha_{j} \widetilde{f}_{m, n, 0}\left(\theta_{j}\right)-\cos 2 \alpha_{j} \widetilde{f}_{m, n, 1}\left(\theta_{j}\right) \\
\widetilde{F}_{m, n, 3}\left(\theta_{j}, \alpha_{j}\right)=\sin 2 \alpha_{j} \widetilde{f}_{m, n, 3}\left(\theta_{j}\right) \\
\widetilde{F}_{m, n, 4}\left(\theta_{j}, \alpha_{j}\right)=\frac{1}{2} \sin ^{2} \alpha_{j} \widetilde{f}_{m, n, 0}\left(\theta_{j}\right)-\sin 2 \alpha_{j} \widetilde{f}_{m, n, 1}\left(\theta_{j}\right) \\
\widetilde{F}_{m, n, 5}\left(\theta_{j}, \alpha_{j}\right)=\cos 2 \alpha_{j} \widetilde{f}_{m, n, 3}\left(\theta_{j}\right) \\
\widetilde{F}_{m, n, 6}\left(\theta_{j}, \alpha_{j}\right)=\cos \alpha_{j} \widetilde{f}_{m, n, 4}\left(\theta_{j}\right) \\
\widetilde{F}_{m, n, 7}\left(\theta_{j}, \alpha_{j}\right)=\sin \alpha_{j} \widetilde{f}_{m, n, 5}\left(\theta_{j}\right) \\
\widetilde{F}_{m, n, 8}\left(\theta_{j}, \alpha_{j}\right)=-\sin \alpha_{j} \widetilde{f}_{m, n, 4}\left(\theta_{j}\right) \\
\widetilde{F}_{m, n, 9}\left(\theta_{j}, \alpha_{j}\right)=\cos \alpha_{j} \widetilde{f}_{m, n, 5}\left(\theta_{j}\right) .
\end{array}\right\}
$$

From (9) follows

$$
\left.\begin{array}{rl}
\widetilde{f}_{m, n, 0}\left(\theta_{j}\right)= & \bar{P}_{n,|m|}\left(\cos \theta_{j}\right), \\
\widetilde{f}_{m, n, 1}\left(\theta_{j}\right)= & \widetilde{a}_{n, m} \bar{P}_{n,|m|-2}\left(\cos \theta_{j}\right)+\widetilde{b}_{n, m} \bar{P}_{n,|m|}\left(\cos \theta_{j}\right) \\
& +\widetilde{c}_{n, m} \bar{P}_{n,|m|+2}\left(\cos \theta_{j}\right), \\
\widetilde{f}_{m, n, 2}\left(\theta_{j}\right)= & -f_{m, n, 0}\left(\theta_{j}\right)+f_{m, n, 1}\left(\theta_{j}\right), \\
\widetilde{f}_{m, n, 3}\left(\theta_{j}\right)= & \widetilde{d}_{n, m} \bar{P}_{n-1,|m|-2}\left(\cos \theta_{j}\right)+\widetilde{g}_{n, m} \bar{P}_{n-1,|m|} \cos \left(\theta_{j}\right) \\
& +\widetilde{h}_{n, m} \bar{P}_{n-1,|m|+2}\left(\cos \theta_{j}\right), \\
\widetilde{f}_{m, n, 4}(\theta)= & \widetilde{\beta}_{n, m} \bar{P}_{n,|m|-1}\left(\cos \theta_{j}\right)+\widetilde{\gamma}_{n, m} \bar{P}_{n,|m|+1}\left(\cos \theta_{j}\right) \\
\widetilde{f}_{m, n, 5}\left(\theta_{j}\right)= & \widetilde{\mu}_{n, m} \bar{P}_{n-1,|m|-1}\left(\cos \theta_{j}\right)+\widetilde{v}_{n, m} \bar{P}_{n-1,|m|+1}\left(\cos \theta_{j}\right) .
\end{array}\right\}
$$

Equations (25) and (26) give

$$
\left.\begin{array}{rl}
\cos \theta_{j} & =\frac{Z_{1 j}}{r_{j}}, \\
r_{j} & =\sqrt{X_{1 j}^{2}+Y_{1 j}^{2}+Z_{1 j}^{2}} . .
\end{array}\right\}
$$

From (30) and (31) we have

$$
\left.\begin{array}{l}
C_{1 j}=Y_{j} \dot{Z}_{j}-Z_{j} \dot{Y}_{j} \\
C_{2 j}=Z_{j} \dot{X}_{j}-X_{j} \dot{Z}_{j} \\
C_{3 j}=X_{j} \dot{Y}_{j}-Y_{j} \dot{X}_{j}
\end{array}\right\}
$$

and

$$
C_{j}=\sqrt{C_{1 j}^{2}+C_{2 j}^{2}+C_{3 j}^{2}} .
$$

From (32) and (24) it is obtained 


$$
\sin \alpha_{j}=\frac{r_{j}}{\sqrt{X_{1 j}^{2}+Y_{1 j}^{2}}} \frac{C_{3 j}}{C_{j}}
$$

and

$$
\cos \alpha_{j}= \pm \sqrt{1-\sin ^{2} \alpha_{j}}
$$

From (28) and (2) follows

$$
\lambda_{j}=\arctan \frac{Y_{1 j}}{X_{1 j}}
$$

and

$$
Q_{m}\left(\lambda_{j}\right)=\left\{\begin{array}{l}
\cos m \lambda_{j}, m \geq 0 \\
\sin |m| \lambda_{j}, m<0 .
\end{array}\right.
$$

In the result, equations (35), (37), (39) - (49) and the constants from Appendix A provide the formulas for computation of the functions of time in the right-hand sides of the basic equations (38).

The procedures of computations can be as follows.

Before evaluating the functions $\bar{F}_{n, m, k}\left(t_{j}\right)$ in the right-hand sides of equations (38), for a fixed $m$ and different $n$ and $k$, the constants are evaluated. The constants entering equations (42) are estimated by the formulas given in Appendix A. The quantities $\sigma_{n}\left(r_{0}\right)$ and $v_{p}$, which also do not depend on $t_{j}$, are found from (35) and (37).

The basic functions of time, $\cos \theta_{j}, \sin \alpha_{j}$ and $\cos \alpha_{j}$, are calculated from equations (43) - (47).

The associated Legendre functions in (42), depending on $\cos \theta_{j}$, are evaluated by recurrent procedures, for a fixed $m$ and all $n$. For this purpose very effective algorithms can be applied which are elaborated by Holmes and Featherstone (2002).

The functions $\tilde{f}_{n, m, k}$ are calculated from (42) and substituted in (41), together with the known $\sin \alpha_{j}$ and $\cos \alpha_{j}$. Then from (41) the functions $\widetilde{F}_{n, m, k}\left(t_{j}\right)$ are evaluated.

The found quantities (35), (40) and (41) are substituted in (39), from where the functions $\bar{F}_{n, m, k}\left(t_{j}\right)$ are estimated.

Finally, from (48) and (49) the functions $Q_{m}\left(\lambda_{j}\right)$ are found.

In the result, all the functions of time in the right-hand sides of Eqs. (38) are evaluated. 


\section{FINAL COMMENTS}

The derived simple expansions for the orbital derivatives contain only two sums and do not contain singular factors. Due to a small eccentricity of the orbit of the GOCE satellite, it is sometimes ignored in the conventional expansions, as well as in the earlier expressions of the authors. In the present expressions the orbit eccentricity and inclination are arbitrary $(0 \leq e<1$ and $0 \leq I \leq \pi)$. The argument of the latitude is also arbitrary, including the values $\omega_{0}=0, \pi / 2, \pi$, which are singular in some conventional expansions of the orbital derivatives.

The final expressions for the orbital derivatives depend neither on the Keplerian elements, no on the spherical coordinates. Instead, they are presented, for the first time, as simple functions of the cartesian coordinates of the satellite and its velocity.

The new expressions may be convenient for processing a huge amount of the gradiometry data when constructing a geopotential model from the GOCE mission data.

The successive steps in the simultaneous implementation of the new expressions for the north-oriented and orbital derivatives of the geopotential can be the same as it is described by Petrovskaya and Vershkov (2006), in Section 6.

The general character of the derived expressions makes possible to apply them not only for the case of the GOCE mission but also for another satellite gradiometry program for any planet.

Acknowledgements. The authors are very grateful to Dr. R. Koop and Dr. J. Bouman for valuable comments and recommendations.

\section{APPENDIX A}

\section{CONSTANTS}

$$
\left.\begin{array}{rl}
\widetilde{a}_{n, m} & =0,|m|=0.1 \\
\widetilde{a}_{n, m} & =\frac{\sqrt{1+\delta_{|m| 2}}}{4(n+1)(n+2)} \sqrt{n^{2}-(|m|-1)^{2}} \sqrt{n+|m|} \sqrt{n-|m|+2}, 2 \leq|m| \leq n \\
\widetilde{b}_{n, m} & =\frac{(n+|m|+1)(n+|m|+2)}{2(|m|+1)(n+1)(n+2)},|m|=0,1 \\
\widetilde{b}_{n, m} & =\frac{n^{2}+m^{2}+3 n+2}{2(n+1)(n+2)}, 2 \leq|m| \leq n \\
\widetilde{c}_{n, m} & =\frac{\sqrt{1+\delta_{|m|, 0}}}{4(n+1)(n+2)} \sqrt{n^{2}-(|m|+1)^{2}} \sqrt{n-|m|} \sqrt{n+|m|+2},|m|=0.1 \\
\widetilde{c}_{n, m} & =\frac{1}{4(n+1)(n+2)} \sqrt{n^{2}-(|m|+1)^{2}} \sqrt{n-|m|} \sqrt{n+|m|+2}, \\
2 & \leq|m| \leq n, n=2,3, \ldots, N
\end{array}\right\}
$$




$$
\begin{aligned}
& \widetilde{d}_{n, m}=0,|m|=0,1 \\
& \widetilde{d}_{n, m}=-\frac{m}{4|m|(n+1)(n+2)} \sqrt{\frac{2 n+1}{2 n-1}} \sqrt{1+\delta_{|m|, 2}} \sqrt{n^{2}-(|m|-1)^{2}} \\
& \times \sqrt{n+|m|} \sqrt{n+|m|-2}, 2 \leq|m| \leq n \\
& \widetilde{g}_{n, 0}=0 \\
& \widetilde{g}_{n, m}=\frac{m}{4|m| \sqrt{n+1}} \sqrt{\frac{2 n+1}{2 n-1}} \sqrt{n-1},|m|=1 \\
& \widetilde{g}_{n, m}=\frac{m}{2(n+1)(n+2)} \sqrt{\frac{2 n+1}{2 n-1}} \sqrt{n+|m|} \sqrt{n-|m|}, 2 \leq|m| \leq n \\
& \tilde{h}_{n, 0}=0 \text {, } \\
& \tilde{h}_{n, m}=\frac{m}{4|m|(n+1) \sqrt{n+2}} \sqrt{\frac{2 n+1}{2 n-1}} \sqrt{n-3} \sqrt{n-2} \sqrt{n-1},|m|=1 \\
& \tilde{h}_{n, m}=\frac{m}{4|m|(n+1) \sqrt{n+2}} \sqrt{\frac{2 n+1}{2 n-1}} \sqrt{n^{2}-(|m|+1)^{2}} \sqrt{n-|m|} \sqrt{n-|m|-2}, \\
& 2 \leq|m| \leq n, n=2,3, \ldots, N \\
& \widetilde{\beta}_{n, 0}=0 \\
& \widetilde{\beta}_{n, m}=\frac{1}{2(n+1)} \sqrt{1+\delta_{|m|, 1}} \sqrt{n+|m|} \sqrt{n-|m|+1}, 1 \leq|m| \leq n \\
& \tilde{\gamma}_{n, 0}=-\sqrt{\frac{n}{2(n+1)}} \\
& \tilde{\gamma}_{n, m}=-\frac{1}{2(n+1)} \sqrt{n-|m|} \sqrt{n+|m|+1}, 1 \leq|m| \leq n, \\
& n=2,3, \ldots, N \\
& \widetilde{\mu}_{n, 0}=0 \\
& \widetilde{\mu}_{n, m}=-\frac{m}{|m|} \frac{1}{2(n+1)} \sqrt{\frac{2 n+1}{2 n-1}} \sqrt{1+\delta_{|m|, 1}} \sqrt{n+|m|} \sqrt{n+|m|-1}, \\
& 1 \leq|m| \leq n \\
& \widetilde{v}_{n, 0}=0 \\
& \widetilde{v}_{n, m}=-\frac{m}{|m|} \frac{1}{2(n+1)} \sqrt{\frac{2 n+1}{2 n-1}} \sqrt{n-|m|} \sqrt{n-|m|-1}, \\
& 1 \leq|m| \leq n, n=2,3, \ldots, N \text {; }
\end{aligned}
$$




$$
\delta_{p, q}=\left\{\begin{array}{l}
1, p=q, \\
0, p \neq q .
\end{array}\right.
$$

\section{APPENDIX B}

\section{DERIVTION OF THE EQUATIONS (21) - (23)}

In Figs. 3, 4 the satellite track azimuth $\alpha$ is presented in connection with the angular variables $\theta, \omega_{0}$ and $I$. The case of $\pi / 2 \leq I \leq \pi$ is considered, corresponding to the GOCE mission, for the north hemisphere. For the other cases similar pictures are also constructed but not presented here.

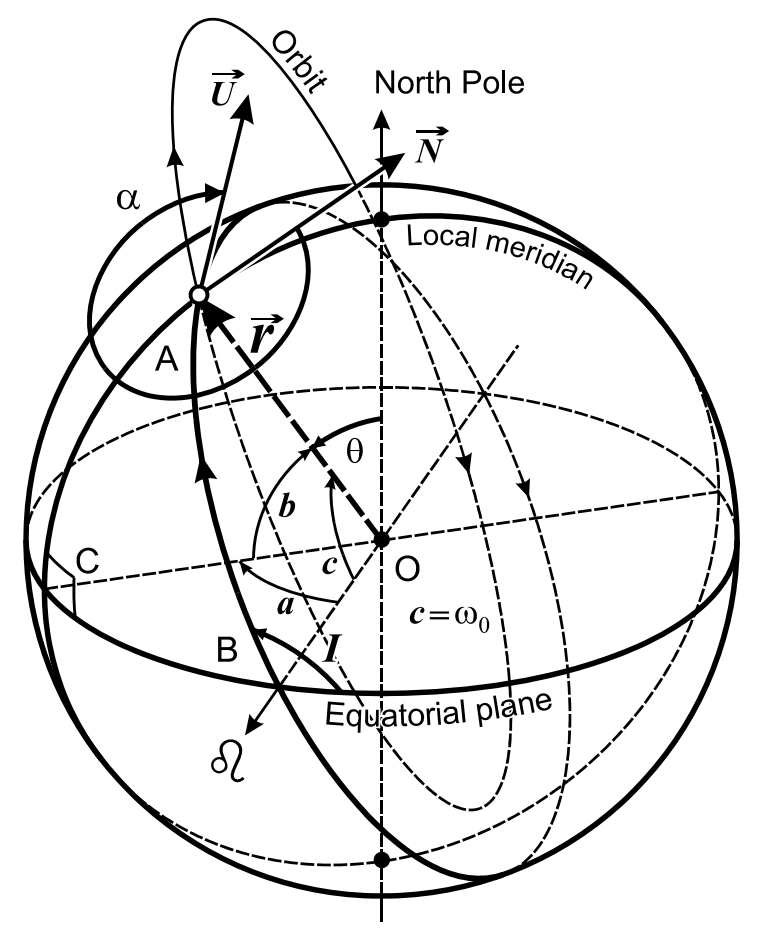

Fig. 3. Satellite track azimuth $\alpha$ for ascending track in the case of $\pi / 2 \leq I \leq \pi$ 


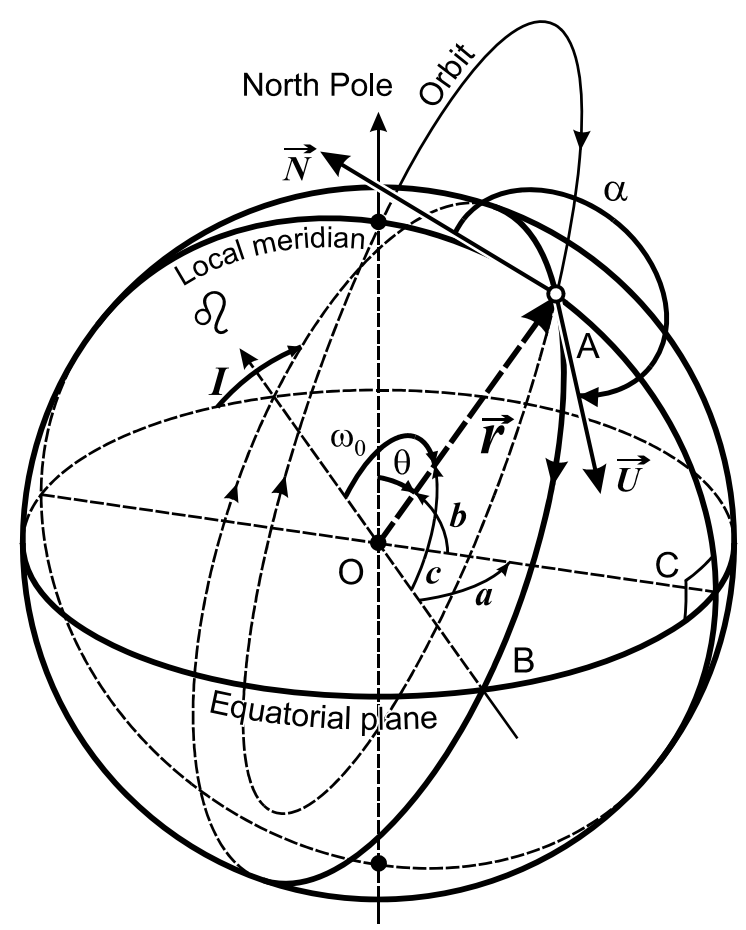

Fig. 4. Satellite track azimuth $\alpha$ for descending track in the case of $\pi / 2 \leq I \leq \pi$

By $O$ the Earth center is denoted. Vector $\vec{U}$ is directed along the axis $u(\vec{U} \perp \vec{r})$ and vector $\vec{N}$ points to the North, tangentially to the local meridian.

Let us consider the spherical triangles $A B C$ in Figs. 3, 4 with the sides $a=C B, b=C A$ and $c=A B$. The arc $A B$ belongs to the section of the sphere of radius $r$ by the orbital plane.

The following relations of the spherical trigonometry between the elements of the spherical triangle $A B C$ are applied

$$
\begin{gathered}
\cos B=-\cos A \cos C+\sin A \sin C \cos b, \\
\frac{\sin b}{\sin B}=\frac{\sin c}{\sin C}, \\
\cos A=-\cos B \cos C+\sin B \sin C \cos a, \\
\cos a=\cos b \cos c+\sin b \sin c \cos A .
\end{gathered}
$$

Since $C=\pi / 2$ then (55) can be written in the form

$$
\sin A=\frac{\cos B}{\cos b} .
$$

From (56) the relations follow

$$
\sin b=\sin B \sin c, \cos b=\sqrt{1-\sin ^{2} B \sin ^{2} c}
$$

and 


$$
\sin c=\frac{\sin b}{\sin B}, \quad \cos c=\sqrt{1-\frac{\sin ^{2} b}{\sin ^{2} B}} .
$$

By inserting the second equation from (60) in (59) we derive the equation

$$
\sin A=\frac{\cos B}{\sqrt{1-\sin ^{2} B \sin ^{2} c}} .
$$

Equations (59) and (62) provide two forms of expressions for $\sin A$.

By substituting (58) in (57) and setting $C=\pi / 2$ we obtain

$$
\cos A=\frac{\sin B \cos b \cos c}{1-\sin b \sin c \sin B} .
$$

From (63), after applying (61) and (60), two forms of expressions are derived for $\cos A$ :

$$
\cos A=\frac{\sqrt{\sin ^{2} B-\sin ^{2} b}}{\cos b}
$$

and

$$
\cos A=\frac{\sin B \cos c}{\sqrt{1-\sin ^{2} B \sin ^{2} c}} .
$$

Let us consider the case, corresponding to Fig. 3.

We have

$$
\begin{aligned}
& \frac{\pi}{2} \leq I \leq \pi, 0 \leq \theta \leq \frac{\pi}{2}, 0 \leq \omega_{0} \leq \frac{\pi}{2}, \frac{3}{2} \pi \leq \alpha \leq 2 \pi ; \\
& A=2 \pi-\alpha, B=\pi-I, C=\frac{\pi}{2}, b=\frac{\pi}{2}-\theta, c=\omega_{0}
\end{aligned}
$$

In (59), (60), (62), (64) and (65) the transfer is performed to the quantities $I, \theta, \omega_{0}, \alpha$ by means of relations (66).

From (60) equations (21) follow. From (62) and (65) we obtain equations (22). On the basis of equations (59) and (64) equations (23) are derived.

Similar derivation was carried out for the case presented in Fig. 4 and for the remaining cases.

\section{REFERENCES}

Ditmar P., Klees R. (2002) A method to compute the Earth's gravity field from SGG/SST data to be acquired by the GOCE satellite. Delft University Press, Delft, Netherlands.

Ditmar P., Klees R., Kostenko F. (2003) Fast and accurate computation of spherical harmonic coefficients from satellite gradiometry data. Journal of Geodesy, Vol. 76, No. 11-12, pp. 690-705.

ESA (2003) ESA’s gravity mission GOCE. BR-209, ESA Publication Division, Netherlands. 
Holmes S. A., Featherstone W. E. (2002) A unified approach to the Clenshaw summation and the recursive computation of very high degree and order normalized associated Legendre functions. Journal of Geodesy, Vol. 76, No. 8, pp. 279-299.

Heiskanen W. A., Moritz H. (1967) Physical Geodesy. Freeman and Company, San Francisco.

Ilk K. H. (1983) Ein Beitrag zur Dynamik ausgedehnter Körper Gravitationswechselwirkung. Deutsche Geodätische Kommission, Reihe C, Heft No. 288, München.

Kaula W. M. (1966) Theory of Satellite Geodesy. Blaisdell Publishing Company, Waltham, Massachusetts.

Klees R., Koop R., Visser P., van den IJssel J. (2000a) Efficient gravity field recovery from GOCE gravity gradient observations. Journal of Geodesy, Vol. 74, pp. 561-571.

Klees R., Koop R., Visser P., van den IJssel J. (2000b) Data analysis for the GOCE mission. International Association of Geodesy Symposia, Vol. 121, (Ed. Schwarz) Geodesy Beyond 2000 - The Challenges of the First Decade, pp. 69-74.

Koop R. (1993) Global gravity field modelling using satellite gravity gradiometry. Publ. Geodesy, New series, No. 38, Netherlands Geodetic Commission, Delft.

Pail R., Plank G. (2002) Assessment of three numerical solution strategies for gravity field recovery from GOCE satellite gravity gradiometry implemented on a parallel platform. Journal of Geodesy, Vol. 76, No. 8, pp. 462-474.

Petrovskaya M. S., Vershkov A. N. (2006) Non-singular expressions of the gravity gradients in the local north-oriented and orbital reference frames. Journal of Geodesy, Vol. 80, No. 3, pp. 117-127.

Reed G. B. (1973) Application of kinematical geodesy for determining the short wave length components of the gravity field by satellite gradiometry. Ohio State University, Dept. of Geod. Sciences, Rep. No. 201, Columbus, Ohio.

Rummel R., Sansò F., van Gelderen M., Koop R., Schrama E., Brovelli M., Migliaccio F., Sacerdote F. (1993) Spherical harmonic analysis of satellite gradiometry. Publ. Geodesy, New Series, No. 39, Netherlands Geodetic Commission, Delft.

Smart W. M. (1953) Celestial Mechanics. Longmans, Green and Co, London - New York Toronto.

Sneeuw N. (1991) Inclination functions. Group Theoretical Background and a Recursive Algorithm, Department of Mathematical and Physical Geodesy, Rep. 91.2, Delft University of Technology.

Sneeuw N. (1992) Representation coefficients and their use in satellite geodesy. Manuscripta Geodaetica, Vol.17, pp. 117-123.

Sneeuw N. (2000) A semi-analytical approach to gravity field analysis from satellite observations. Reihe C, Heft Nr. 527, Deutsche Geodätische Kommission, München. 
Sneeuw N., Dorobantu R., Gerlach C., Müller J., Oberndorfer H., Rummel R., Koop R., Visser P., Hoyng P., Selig A., Smit M. (2001) Simulation of the GOCE gravity field mission. In: Benciolini B. (ed.) IV Hotine-Marussi Symposium on Mathematical Geodesy. IAG Symposia Vol. 122, Springer, Berlin - Heidelberg - New York, pp. 14-20.

Vermeer M. (1990) Observable quantities in satellite gradiometry. Bull. Géod., Vol. 64, pp. $347-361$.

Received: 2007-03-26,

Reviewed: 2007-08-26, by J. Bouman and R. Koop,

Accepted: 2007-10-30. 